\title{
A Distributed Fault-Tolerant MPEG-7 Retrieval Scheme Based on Small World Theory
}

\author{
Panagiotis Androutsos, Member, IEEE, Dimitrios Androutsos, Senior Member, IEEE, and \\ A. N. Venetsanopoulos, Fellow, IEEE
}

\begin{abstract}
A small world search agent employing peer-to-peer (P2P) concepts borrowed from sociology is employed for performing image retrievals in a small world distributed media index. The Small World Indexing Method (SWIM) allows for a highly networked architecture where index information does not exist as a separate entity on a specific server, but rather is stored within the actual media objects themselves. Since each media object is only responsible for a small portion of the overall index, the loss of portions of the overall network (data objects) accounts for only a small degradation in the overall retrieval performance. Building upon previous work, the graceful degradation which is provided by the SWIM system is addressed here for retrievals which are performed using small world user agents on a large set of MPEG-7 described images.
\end{abstract}

Index Terms-Distributed, fault-tolerant, indexing, MPEG-7, retrieval, small world.

\section{INTRODUCTION}

$\mathbf{I}$ $\mathrm{N}$ the last five years, media indexing has fulfilled many of the dreams and concepts which were originally envisioned by the developers of IBM's QBIC [1]. The MPEG-7 standard is only one of these huge steps which has unified content-based indexing and brought previously disparate systems closer together by outlining specific rules for performing media description. At the same time, however, content-based indexing has also ventured into unforseen areas, more specifically that of distributed computing. Both the COMPASS [2] and DISCOVIR [3] systems have addressed this issue with some success using de-centralized and peer-to-peer concepts related to distributed computing initiatives like CHORD [4]. The great advantage of using distributed approaches to performing any computing task lies in the fact that distributed systems exhibit a graceful degradation in system performance as components experience failure at varying levels. This is to say that unlike centralized approaches, failure of one component does not result in a complete system failure. Rather, under a distributed paradigm, when a component fails, the overall system remains functional, but only partially so. Partial decreases in system performance are imperative in

Manuscript received March 8, 2005; revised October 9, 2005. The associate editor coordinating the review of this manuscript and approving it for publication was Prof. Rynson W. H. Lau.

P. Androutsos is with Dominion Voting Systems, Toronto, ON M6K 3E8 Canada (e-mail: peter@dvscorp.com).

D. Androutsos is with the Department of Electrical and Computer Engineering, Ryerson University, Toronto, ON M5B 2K3 Canada (e-mail: dimitri@ee.ryerson.ca).

A. N. Venetsanopoulos is with the Edward S. Rogers Sr. Department of Electrical and Computer Engineering, University of Toronto, Toronto ON M5S 3G4 Canada (e-mail: anv@dsp.toronto.edu).

Digital Object Identifier 10.1109/TMM.2005.864276 many computing situations where a service is provided. Since a system-wide failure means lack of service, and thus lack of income, a degraded system results in slightly worse or partial service, and thus a reduction in revenue. This results in a higher level of survivability for the overall system, but comes at the cost of increased overhead and complexity.

The fault-tolerance investigation presented in this paper employs Small World thoery to create a Small World Indexing Mine (SWIM) [5] along with an image database processed using standard MPEG-7 descriptors. This paper's organization is as follows. Section II outlines the underlying theory of the SWIM system and provides an explanation of its background, development and overall function. Following this, the retrieval agent used in the SWIM environment is discussed and then Section III explains the experiments performed and the results achieved which illustrate the system's graceful performance degradation. The paper then concludes with summarizing closing statements.

\section{SMALl WorldS AND SMAll WorLd INDEXING}

Early studies on the sociological phenomenon known as the "small world" effect were performed by Pool and Kochen [6], yet it was Milgram who first put social acquaintance network theory into action and arrived at the conclusion that the typical number of hops or degrees of separation between any two people was approximately equal to six [7]. Often referred to as the "six degrees of separation" phenomenon, the Small World Effect has become so well-known that it was also popularized in the 1990s by a play [8], and even by a game known as "The Six Degrees of Kevin Bacon" [9], [10]. Various studies have shown that the World Wide Web (WWW) also demonstrates Small World characteristics [11]-[13]. With over 2 billion pages, it is extremely interesting that the WWW only has an average diameter from between 16 and 20 [14], meaning that assuming knowledge of an ideal path, almost any webpage can be reached from nearly any other with an average of seventeen clicks of the mouse. Thus, despite the seemingly chaotic and unregulated growth of the web, there does exist some form of self-organization resulting from the way in which webpages (or alternatively URLs) hyperlink to each other. This self-organization arises from the linking of webpages sharing similar subject matter (as is the case with web communities) via extremely large referral and news sites such as Google, Yahoo, BBCNews, and CNN, or by random connections such as "sites of the day." Shown in Fig. 1(a) is a possible hyperlinking topology consisting of ten webpages for members of a university research lab. An interesting news item (A) featuring a specific university provides a link to the school in question (B). The university, then may subsequently provide a link 


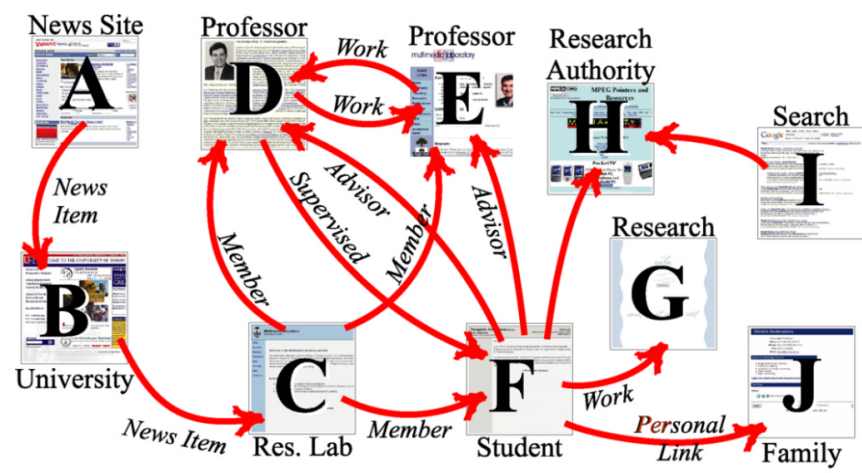

(a)

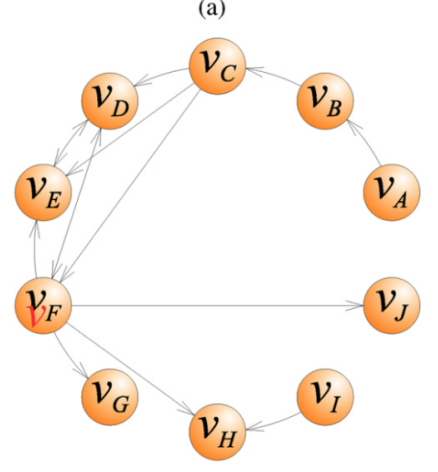

(b)

Fig. 1. (a) Ten webpages hyperlinking to each other using directed links where each edge exists as a result of a specific relationship or shared interest. (b) The same topology represented as an equivalent digraph. (Color version available online at http://ieeexplore.ieee.org)

to a specific research lab (C). Consisting of a number of professors and students, the page from the research lab points to its members $(\mathrm{D}, \mathrm{E}, \mathrm{F})$ who in turn have personal pages pointing to each other, topics of interest, relatives $(\mathrm{G}, \mathrm{H}, \mathrm{I}, \mathrm{J})$ etc. This (albeit simplified) topology illustrates the complex set of relationships which connect URLs according to content in a fashion mirroring social acquaintance networks which may connect people according to shared or similar interests. Notwithstanding, in a sociological context, shared interests are bidirectional, meaning that if one person is connected to an acquaintance because of a common interest, then the reverse is also true. This is not the case for the WWW, where directed links to related URLs do not require target URLs to point back to their referrers.

The asymmetric nature of these links has some important implications, the most obvious of which is the fact that a path between any two given URLs is not necessarily (and usually not at all) bidirectional [15]. Secondly, the distinction which needs to be made between links that are incident to and incident from specific webpages leads to difficulties in creating a generalized framework for effectively modeling the World Wide Web's behavior without treating incoming and outgoing edges separately. This implies a network characterization which necessarily decouples incoming and outgoing degree distributions [16].

From a more scientific perspective, however, the Small World problem was recently analyzed and modeled by Watts and Strogatz [17], leading to a refuelling of interest in these topics. In this section, a brief outline of small world theory will be discussed along with some specifics on the inner workings of small world distributed indexes.



(a)



(b)

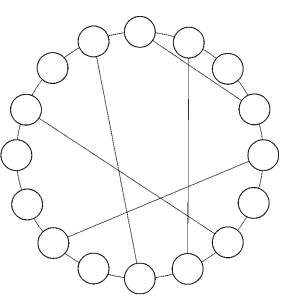

(c)
Fig. 2. (a) The 1-lattice ring of $N$ nodes can be merged with an (b) Erdós-Rényí random graph with edge probability $p$ to model a small world network. (c) The Newman-Watts model with edge probability $p \ll 1$ merges a lattice with a random graph by adding random links between lattice nodes.

\section{A. Small World Models}

Deeply steeped in graph theory, the concepts behind social network analysis and small worlds, are most simply represented in the form of graphs. A one-dimensional 1-lattice as shown in Fig. 2(a) is required for the small world model considered here. Such an undirected graph is an $N$-node network where edges only exist between nearest neighbors.

This topology is highly regular, unlike the Erdös-Rényí random graph of Fig. 2(b) where any two vertices $v_{i}$ and $v_{j}$ may be connected with probability $p$ [18]. The Newman-Watts small world model [19] combines the aforementioned lattice topology with a random graph to represent a small world network. The necessity for such models stems from the fact that random graphs are sometimes limited in their applicability to the real world. This is because unlike random graphs, real world networks do not always follow Poisson node degree distributions [20], and also because discrepancies exist between the clustering coefficients of random graph models and real measurements [21].

\section{B. SWIM}

The small world paradigm is employed here to perform distributed indexing of MPEG-7 data through the local storing of peer node description data. The MPEG-7 descriptions employed here is the color structure descriptor [22] because of its fixed size and convenience, but this choice is ultimately a matter of choice depending on the particular application or feature that needs to be considered. The Small World Image Miner (SWIM) creates a directed small world network of images which are connected according to MPEG-7 descriptor similarities. The SWIM system attempts to mimic the way humans perform referrals and introductions between acquaintances by having each image in the SWIM network store the descriptions of its most similar peers. This is similar to how humans retain descriptions about the members of their acquaintance network which enables them to intelligently act as proxies and make introductions between their respective unrelated acquaintance according to their perceived characteristics, interests and/or traits. Under this small world concept, images must become active elements within the index, storing descriptor data about their most similar peers as well as constantly interacting with new images and search agents by performing distance calculations and acting as referrers to new or more similar nodes. In addition to specifically addressing multimedia indexing, this system is different than the distributed approach used by $C H O R D$ [4] as no hash 
function is used for distributing and locating data, and searches are performed using a decision-based agent according to local node data descriptions. Although it exhibits some similarity to the DISCOVIR system [3], SWIM permits multiple levels of MPEG-7 descriptor data, locally stores descriptor data at every node, and promotes searching through the exclusive use of directed links. In addition, unlike DISCOVIR, each peer in the SWIM framework is permitted to have its own distinct set of peer connections, and is not limited by peer connections that are dictated by its host computer. Lastly, and most importantly, it is critical to remember that in the SWIM model, the index becomes completely distributed throughout the actual media objects which it indexes. This results in a complete re-evaluation of the concept of an index acting as a representative of a group of images; in the SWIM model, the index and the data it represents merge and become one.

SWIM networks function under a model that very closely resembles that proposed by Newman and Watts, but with some fundamental assumptions and modifications. First, a network with $N$ nodes is initially assumed. Secondly, all connections within the SWIM network are directed in nature in a fashion resembling the structure of the WWW. This is in contrast to the models in [17] and [19] which exclusively use undirected edges. Specifically, the SWIM system uses an underlying directed $k$-lattice together with a directed pseudo-random graph $^{1}$ in an $N$-node network. In order to avoid incorrectly referring to this graph as "random", it is called a Similarity Graph, and denoted as $\mathbf{G}_{S}$. The term pseudo-random is employed here because unlike the Newman-Watts model, the SWIM network does not establish random connections between nodes according to a set probability value $p$. Instead, connections are established according to a particular external distance measure $D$ operating on data stored locally by each node, and obtained via descriptor $d$. The distance measure $D$ refers to a function which is used to calculate the error between two sets of data. As mentioned, these data sets are generated by applying a descriptor $d$ to a vertex in order to extract node-specific information. Here, for a particular descriptor, these data sets take the form of a description vector $\vec{d}$. Thus, for two vertices $v_{1}$ and $v_{2}$ which are described by the vectors $\overrightarrow{d_{1}}$ and $\overrightarrow{d_{2}}$ obtained using descriptor $d$, the distance (error) between them is denoted as $D\left(\vec{d}_{1}, \overrightarrow{d_{2}}\right)$. Many different descriptions can be generated yet the most straightforward is a descriptor which generates the $x-y$ coordinates of each vertex; a two-dimensional description vector. The edges shown therein could then be established by calculating all the Euclidean distances between all descriptions, and only making connections between vertices with the smallest distances between them (i.e., the vertices with similar spatial position on the 2-D plane).

Using conventional graph notation, a similarity graph $\mathbf{G}_{S}$ for a given distance measure $D$ and descriptor $d$, can be expressed with a vertex and edge set pair as:

$$
\mathbf{G}_{S}=\left(\mathbf{V}_{N}, \mathbf{E}_{S}(F, d, D)\right)
$$

where $\mathbf{V}_{N}$ denotes the set of vertices $\left\{v_{1}, v_{2}, \ldots, v_{N}\right\}$ and the edge set $\mathbf{E}_{S}$ is dependent on the number of Small World

\footnotetext{
${ }^{1}$ This graph is random only in its appearance.
}

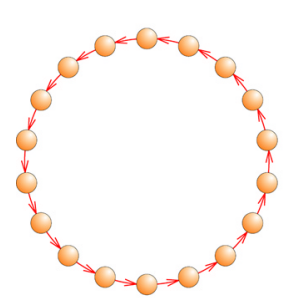

(a)



(b)

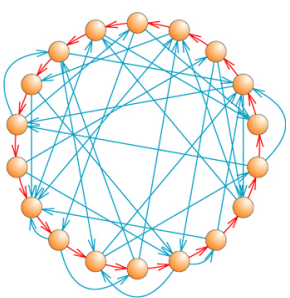

(c)
Fig. 3. (a) Sixteen-node, directed, 1-lattice ring forming the weak graph $\mathbf{G}_{\mathbf{W}}$. (b) The corresponding similarity graph $\mathbf{G}_{S}$ with $F=2$ using a given descriptor and distance measure. (c) The overall SWIM graph made by combining $\mathbf{G}_{S}$ and $\mathbf{G}_{W}$. (Color version available online at http://ieeexplore.iee.org)

peers $F$, the particular descriptor $d$, and the distance measure $D$ employed.

In contrast to $\mathbf{G}_{S}$, the directed $k$-lattice ring component of the SWIM model creates connections by joining nodes to their $k$ successors with a periodic boundary condition. Since these directed connections are established independently of distance measure and descriptor, they create a weak graph (i.e., not based on similarity) which is denoted $\mathbf{G}_{W}$, and is defined using (2) and (3)

$$
\begin{aligned}
\mathbf{G}_{W} & =\left(\mathbf{V}_{N}, \mathbf{E}_{W}(k)\right) \\
\mathbf{E}_{W}(k) & =\left\{\forall\left(v_{i}, v_{j}\right) \mid \begin{array}{rl}
\text { if } i<N-\ell: & j=i+\ell \\
\text { else }: & j=i+\ell-N
\end{array}\right\}
\end{aligned}
$$

where $i, j=1,2, \ldots, N$ and $\ell=1,2, \ldots, k$. Fig. 3(a) and (b), respectively, illustrate a typical SWIM network's similarity and weak graphs for a given distance measure and descriptor where $N=16, k=1$, and $F=2$, while Fig. 3(c) shows their union in forming an overall SWIM network. When viewing these graphs, the highly regular structure of $\mathbf{G}_{W}$ is immediately apparent, while the connections contained in $\mathbf{G}_{S}$ seem almost random (which is why the term pseudo-random is employed).

It is important to note here that a given similarity graph $\mathbf{G}_{S}$ is associated with a specific descriptor and distance measure. The use of a different descriptor for describing the data represented by the network nodes produces a different similarity graph. Furthermore, the use of a different distance measure $D$ also results in a different $\mathbf{G}_{S}$. By comparison, the weak graph $\mathbf{G}_{W}$ is neither dependent on the distance measure employed or the descriptor chosen for describing the node data. Instead, $\mathbf{G}_{W}$ is only dependent on the order in which nodes are introduced into the network, and as additional description and distance measure layers are incorporated into the system, it remains fixed for a given value of the lattice parameter $k$.

Furthermore, because of "cliquish" node behavior observable for particular combinations of $d$ and $D$, it is often possible that a resulting similarity graph $\mathbf{G}_{S}(d, D)$ can become fragmented, resulting in pairs of vertices which cannot be connected via a finite path $\mathbf{P}$. This is where the importance of the weak graph becomes evident, as it creates a ring containing the absolute minimum number of edges for guaranteeing that the graph is not disconnected in any way. Had these graphs been mapped onto the space defined by the descriptor $d$ as opposed to a simple arrangement on the Cartesian plane, the resulting topologies would have 
exhibited high localization of connected entities for the similarity graph $\mathbf{G}_{S}$, while depicting seemingly random connections between elements in $\mathbf{G}_{W}$. Still, despite the improvement in the depiction of node clustering that would result for the (relatively more important) similarity graph, the space emphasizing the regularity of $\mathbf{G}_{W}$ is preferred. This choice is made due to the difficulty that is associated with depicting the relative positions of vertices in descriptor spaces with dimensionalities greater than two.

In both the Watts-Strogatz and Newman-Watts Small World models, random graph edges are used as shortcuts between nodes to bridge vertices in localities of an underlying lattice structure. These models can be interpreted using the concepts of a similarity graph and a weak graph where a node's description $\left(d_{C C}\right)$ is simply its Cartesian coordinates, and the distance measure $\left(D_{E D}\right)$ employed is the Euclidean distance. Taking the Newman-Watts model which adds random links between nodes to the underlying lattice as an example, the underlying lattice acts as the model's similarity graph $\mathbf{G}_{S}=\left(\mathbf{V}, \mathbf{E}_{S}\right)$ where the edge set $\mathbf{E}_{S}=\left\{\mathbf{E}_{S}(i)\right\}$ and

$$
\left.\mathbf{E}_{S}(i)=\left\{\min _{F}\left\{D\left(d\left(v_{i}\right) d\left(v_{j}\right)\right)\right\}\right)\right\}
$$

for all $i=1,2, \ldots N_{V}, v_{i}, v_{j} \in \mathbf{V}, v_{i} \neq v_{j}$ and where duplicate edges are ignored. In (4), each vertex $v_{i}$ has associated with it a set of $F$ edges which are incident upon it and connect it to $F$ peers. Thus, $F$ is equivalent to the lattice parameter $k$. The edges $\mathbf{E}_{W}$ in the equivalent weak graph for the Newman-Watts model would be randomly generated according to a probability $p$.

Now, the parallel between the NW-model and the SWIM model can be seen. In the NW-model, the underlying lattice is used to spatially connect proximal vertices, while the added random graph component acts to provide shortcuts between vertex groupings. In contrast, the SWIM model employs an $F$-peer-per-vertex similarity graph to connect vertices exhibiting high similarity according to a given descriptor-distance measure combination. In addition to ensuring connectedness between vertices, the weak graph of the SWIM model serves to provide shortcuts. These shortcuts are pivotal for vertex neighborhoods since they provide highly clustered cliques of nodes access to other, unrelated clusters that are also tightly knit in their own rights. As explained in both [23] and [24], the strength of these weak ties plays a critical role in the structure of social networks and Small World theory since it enables the members of a particular social group to have access to members of another.

Continuing, a generalized SWIM graph $\mathbf{G}_{S W}$ can now be formalized by combining the expressions of (1) and (2) as

$$
\begin{aligned}
\mathbf{G}_{S W} & =\left(\mathbf{V}_{N}, \mathbf{E}_{W}(k) \bigcup \mathbf{E}_{S}(F, d, D)\right) \\
& =\left(\mathbf{V}_{N}, \mathbf{E}_{O}\right) .
\end{aligned}
$$

Fig. 4 illustrates the above equation, where $\mathbf{G}$ consists of the singular weak graph $\mathbf{G}_{W}$ for a fixed value of $k$, along with the set $\left\{\mathbf{G}_{S}\right\}=\left\{\mathbf{G}_{S}\left(d_{1}\right) \mathbf{G}_{S}\left(d_{2}\right) \ldots, \mathbf{G}_{S}\left(d_{N_{d}}\right)\right\}$ which represents multiple layers of distinct similarity graphs. Since some distance measures are not always applicable

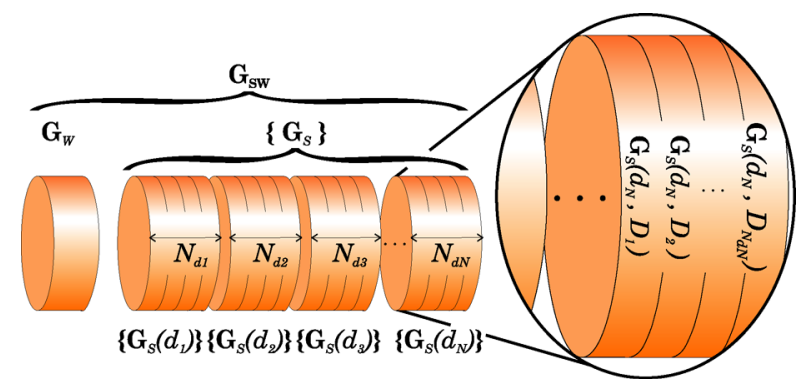

Fig. 4. Generalized SWIM graph for fixed $F$ consisting of the weak graph $\mathbf{G}_{W}(\mathrm{k}=1)$ and the set of similarity graphs $\left\{\mathbf{G}_{S}\right\}$. Each $\left\{\mathbf{G}_{S}\left(d_{i}\right)\right\}$ is further made up of $N_{d_{i}}$ distance measure-specific graphs $\mathbf{G}_{S}\left(d_{i}, D_{j}\right)$. (Color version available online at http://ieeexplore.ieee.org)

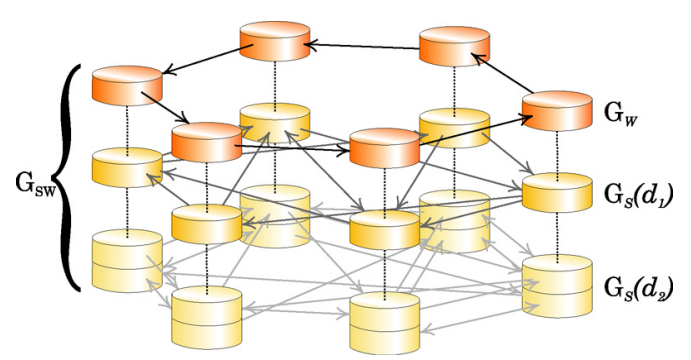

Fig. 5. Overall SWIM graph $\mathbf{G}$ made up of multiple subgraph layers for a fixed number of peers $F=2$ and lattice parameter $k=1$ on six vertices. The layer corresponding to descriptor $d_{1}$ has only one associated distance measure, while $d_{2}$ employs two. (Color version available online at http://ieeexplore.ieee.org)

or admissible for specific descriptors (i.e., it is invalid to apply a Euclidean distance to a non-Euclidean space) [25], [26], the number of distance measures $N_{D_{i}}$ used for a particular descriptor $d_{i}$ can vary. This consequently results in differences in the size of each set of similarity graphs $\left\{\mathbf{G}_{S}\left(d_{i}\right)\right\}=\left\{\mathbf{G}_{S}\left(d_{i}, D_{1}\right) \mathbf{G}_{S}\left(d_{i}, D_{2}\right) \ldots, \mathbf{G}_{S}\left(d_{i}, D_{N_{D_{i}}}\right)\right\}$.

With the knowledge that $\mathbf{G}_{S W}$ can consist of many layers of different similarity graphs, the stacked structure for the overall graph topology can be visualized. Fig. 5 illustrates an example of a particular realization of $\mathbf{G}_{S W}$ using the values $N=6$, $k=1$ and $F=2$, and distinct descriptors $d_{1}$ and $d_{2}$ each respectively employing one and two distance measures.

For a clearer understanding, the sociological standpoint can be taken in order to illustrate the function of the SWIM. Specifically, the Small World Indexing Model mirrors the way people retain knowledge about their own particular circle of friends and acquaintances. Usually, two people are friends or acquaintances due to some common bond. This bond may be related to a common friend or occupation, a shared interest, similar tastes, etc. Every person mentally keeps track of their own descriptions of how or who their friends and contacts are, partially according to these descriptions. These descriptions of specific peers which are retained by every member of a social network form the basis of the SWIM framework. A simplistic scenario is shown in Fig. 6, which illustrates a stranger being introduced to one of a group of three friends. In (a), Rita is introduced to Tom, and they find that they do not share common features (color and shape). As shown in (b), Tom thus refers Rita to his friends Mary and John, whom he knows share something in common with her (shape and color respectively). 


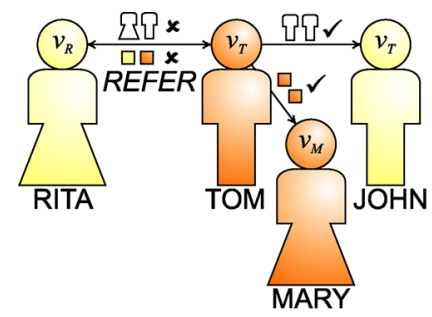

(a)

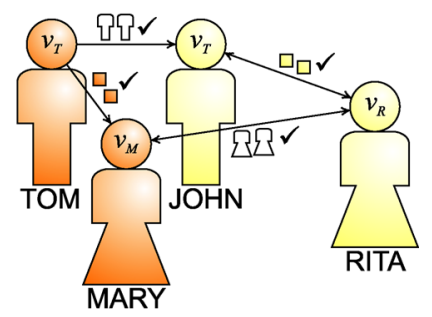

(b)
Fig. 6. In (a), Rita is introduced to Tom with whom she shares no common features. In (b), Tom thus refers Rita to his friends Mary and John, who he knows share common features with her (similar shape and color respectively). (Color version available online at http://ieeexplore.ieee.org)

Making the jump from human social networks to images is not very difficult at this point, since images can be related to each other using image-specific features and descriptions. As long as the images are made into SWIM nodes, they can actively and intelligently interact with each other to dynamically create a SWIM network. It is the mapping of this social networking behavior of performing referrals based on a standardized set of descriptions that allows nodes within a SWIM network are able to actively find other nodes sharing similar characteristics.

\section{SWIM Nodes}

For a given similarity graph within a SWIM network, each node must locally store descriptor data about itself, as well as the actual data $I_{i}$ of the object which it represents. More importantly, however, each node must also keep track of the descriptions for its $F$ peers in $\mathbf{G}_{S}$. This retention of peer information necessitates that each node become a much larger entity which can store a range of descriptions both about itself and its peers, as well as store link information for enabling the peer referral behavior discussed previously. The compartmentalized nature of each SWIM node is illustrated in Fig. 7 which depicts multiple descriptor layers.

As shown in Fig. 7, each SWIMage consists of a top-level interfacing element which provides interaction between other SWIM network elements. In addition, multiple description layers which store self and peer MPEG-7 descriptor data can be seen. Similar to the Newman-Watts model [19], a SWIM network consists of a regular lattice merged with what seems like a random graph. Each SWIM network layer establishes $F$ directed nearest-neighbor links (friends) between vertices using an external distance measure in the creation of its Similarity Graph, $G_{S \ell}$. A single, directed weak link between successive nodes is used to generate the Weak Graph, $G_{W}$. These two components are illustrated in Fig. 8(a) and (b).

\section{Incremental SWIM Graph Growth}

It is neither realistic nor practical to assume that all nodes of interest are available for an a-priori analysis in creating a steady-state small-world network. On the contrary, no assumptions can be made about the data represented by each SWIM node. Moreover, because truly distributed systems by definition require a decentralized approach for all aspects of system operation, the control involved within a Small World indexing framework for facilitating the incorporation of new index elements must also be decentralized in nature. To this end, an incremental

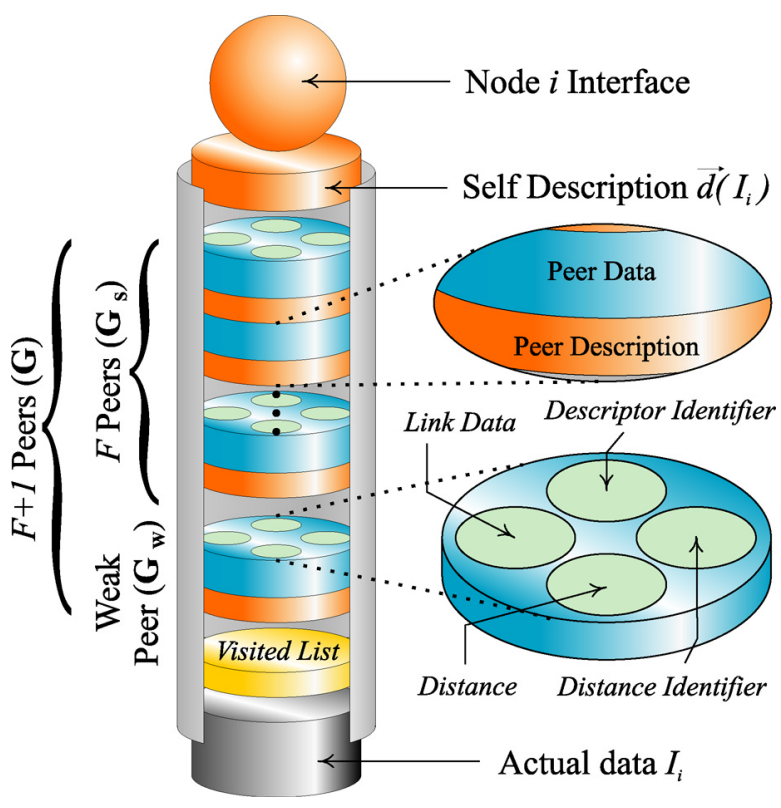

Fig. 7. In addition to actual pixel data, a SWIMage consists of a set description layers and an interfacing for communication between nodes. Each description layer stores peer description data vectors as well as the actual peer data and directed links to similar SWIMages and similarity/distance values. Each node is responsible for one weak peer $G_{W}$ and $F$ peers established according to image similarity $G_{S}$.

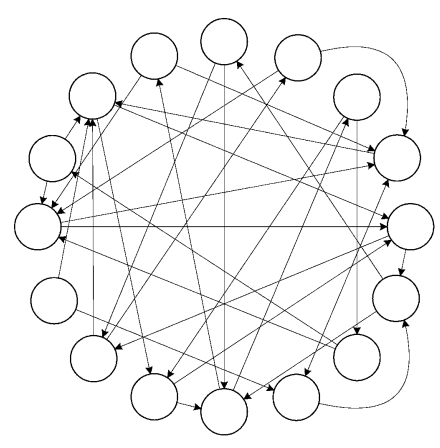

(a)

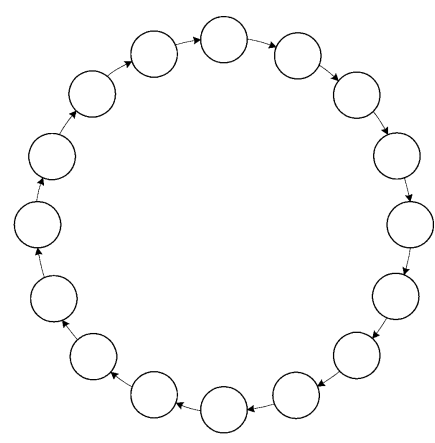

(b)
Fig. 8. Sixteen-node SWIM network. Connections are made (a) from each node to $F=2$ friends according to an external similarity metric to create $G_{S \ell}$ and (b) between successive nodes to create $G_{W}$.

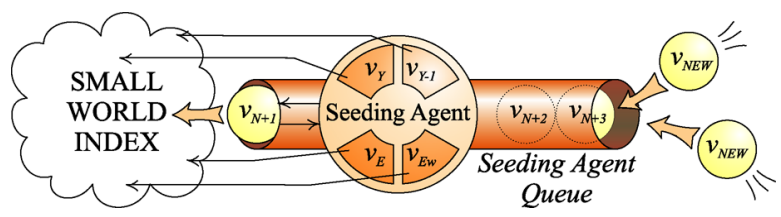

Fig. 9. Seeding agent performs initial introductions between the SWIM network's youngest nodes $\left(v_{Y}, v_{E}, v_{E w}\right.$, etc.) and queued up nodes (e.g., $\left.v_{N+1}, v_{N E W}\right)$. The seeding agent also maintains connectivity within the overall SWIM graph by preserving the integrity of the weak graph's ring structure through tracking of recently introduced vertices. (Color version available online at http://ieeexplore.ieee.org)

SWIM graph growth algorithm is employed where new nodes enter the SWIM network via an insertion point determined by a seeding agent. As illustrated in Fig. 9, this seeding agent also acts to ensure connectivity in the SWIM network by appropriately incorporating them into the underlying weak graph (lattice). The seeding agent achieves this by keeping track of the 
TABLE I

VERTEX-VERTEX DistANCE MATRIX FOR THE EXAMPLE OF FIG. 10

\begin{tabular}{c||c|c|c|c|c}
\hline & $v_{1}$ & $v_{2}$ & $v_{3}$ & $v_{4}$ & $v_{5}$ \\
\hline \hline$v_{1}$ & - & 0.20 & 0.60 & 0.40 & 0.35 \\
\hline$v_{2}$ & 0.20 & - & 0.90 & 0.30 & 0.05 \\
\hline$v_{3}$ & 0.60 & 0.90 & - & 0.65 & 0.50 \\
\hline$v_{4}$ & 0.40 & 0.30 & 0.65 & - & 0.70 \\
\hline$v_{5}$ & 0.35 & 0.05 & 0.50 & 0.70 & - \\
\hline
\end{tabular}

two youngest nodes $v_{Y}$ and $v_{Y-1}$, as well as the most recently established node $v_{E}$ and the node to which it weakly links $v_{E w}$. These particular nodes are tracked for the following reasons: the youngest node $v_{Y}$ in the network is needed in order to properly keep track of maximum number of nodes in the network (assuming no deletions) and the most recently established node $v_{E}$ along with the node which points to it $v_{E w}$ are used for inserting the new node into the SWIM network to maintain connectivity. This places upon the youngest network elements the onus of having to initially deal with new incoming nodes.

Upon being issued an insertion point (i.e., the youngest established network element) a newly incorporated node is subsequently provided with a continuous stream of new peer referrals before finding a "steady-state" within the Small World. This steady-state can be defined as the point at which $v_{N=1}$ no longer has any unexplored nodes left to visit (i.e., it has exhaustively, visited all network elements in the Small World) or when $v_{N+1}$ has visited a predefined threshold number of nodes $N_{t}$. Beyond this point, the node is referred to as established, meaning that it has traversed enough peers in the network to effectively act as a primary referrer for future incoming nodes as well as to sufficiently randomize the initial starting clique of vertices responsible for directing future nodes to their respective self-similar peer set.

The algorithm for inserting new nodes is best explained through the use of a concrete example consisting of a single descriptor, a single distance measure, and a small number of vertices. Table I provides a set of randomly generated vertex-vertex distances used for the example shown in Fig. 10 involving a SWIM network having $N=4, F=2$ and $k=1$. The ultimate goal is the incorporation of the new node $v_{5}$ into the SWIM network by having it visit all nodes in the network and ultimately determine its peer set $\boldsymbol{\Phi}_{5}$. Node $v_{5}$ begins by querying the seeding agent which provides an initial introduction into the system [Fig. 10(a)]. The seeding agent first instructs $v_{3}$ to replace its weak link to $v_{4}$ with $v_{5}$, and then instructs $v_{5}$ to weakly link with $v_{4}$ and contact $v_{3}$ for subsequent node referrals (this represents the initial introduction into the SWIM network where $v_{3}$ is assumed to be "established"). This gives the new vertex a position inside the weak graph $\mathbf{G}_{W}$, and knowledge about node $v_{3}$. In Fig. 10(b), $v_{5}$ occupies a vertex position within the weak graph $\mathbf{G}_{W}$ and has knowledge about $v_{3}$, while in (c) $v_{5}$ establishes its first nonnull peer and is also informed of $v_{3}$ 's peer nodes $\left(v_{1}\right.$ and $\left.v_{4}^{2}\right)$. More importantly, node $v_{3}$ updates its peer set by eliminating its existing link to $v_{4}$ and replacing it with a link to $v_{5}$. Node $v_{5}$ continues by this

\footnotetext{
${ }^{2}$ It is worthwhile to note that as with all nodes that are queued up for visitation using this algorithm, the nodes $v_{1}$ and $v_{4}$ are ordered according to their similarity to $v_{5}$, meaning that highly similar nodes are visited first.
}

process of visiting queued up nodes and obtaining additional peer information. The final state of the incremental growth algorithm example is shown in Fig. 10(e) where $v_{5}$ has no more queued nodes left to visit.

The example is also very illustrative of the very important fact that only newly introduced vertices actively traverse the existing SWIM network to enable the updating of peer links. Old nodes, however, are not required to perform this task, and as shown in the example, new nodes actively find them through peer referrals while attempting to incorporate themselves into the network. The SWIM nodes, can thus exist on completely independent hosts which facilitate these referrals by merely providing network access and resources.

\section{E. SWIM Retrieval Agent Search Algorithm}

Queries made to the SWIM index are achieved with a user agent-based retrieval scheme. This technique functions in a manner that is similar to the incremental SWIM graph growth algorithm outlined in the preceding subsection, as it uses referrals from existing SWIM nodes in order to direct itself to nodes exhibiting higher similarity to query criteria. Mirroring the process of self-organization of SWIM nodes through peer referrals, SWIM agents are inserted into the small world network at particular nodes (images) and hop from image to image through SWIM node referrals. Performed locally by the agent itself, these referrals which direct the agent to successive nodes are determined by comparing query data with each visited node's descriptions, and updating a running list of most attractive nodes to be visited.

To illustrate the algorithm, the simple SWIM network of Fig. 11(a)-(d) is provided. Therein, four SWIM nodes are interconnected according to the distance/incidence matrix shown in Table II, where for simplicity, $F=1$ and only a single descriptor is employed.

In Fig. 11, the agent begins by being inserted into the SWIM network at a seed node $v_{1}$. The agent then measures the distance between its query data and the description data of node $v_{1}$. Since $v_{1}$ is the first node visited by the agent, both a pointer (link) to $v_{1}$ and its descriptor distance are stored in the agent's retrieval link $R_{A}$. After $v_{1}$ is marked as already visited, the agent obtains node $v_{1}$ 's peer information in knowledge of $v_{2}$ (weak peer) and $v_{3}$. These agents are inserted into a target queue (i.e., nodes to visit) according to their descriptor distance to the agent. The agent then proceeds to hop to the next most similar node in its target queue [Fig. 11(b)]. This process continues until the agent has no nodes left to visit.

\section{RETRIEVAL RESILIENCY}

Each image exists as its own entity in the SWIM network, and as explained, maintains links to peer nodes, performs referrals and actively participates in facilitating growth and search within the SWIM index. Yet, each SWIM node must somehow reside on a computer system which hosts it.

Each SWIM node behaves as its own client and server within the peer-to-peer SWIM network, residing on its host computer, which at the same time may host any number of other SWIM nodes as well. So, the actual physical location of SWIM nodes 


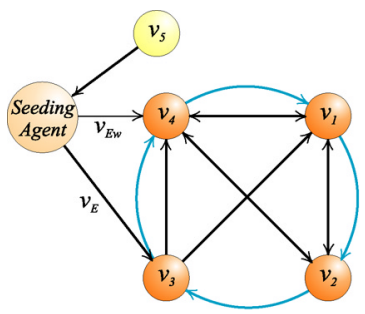

Visited: -

To Visit: -

$\boldsymbol{O}_{\mathrm{D}}=(-,-) \boldsymbol{O}_{\mathrm{d}}=(-,-)$

(a)

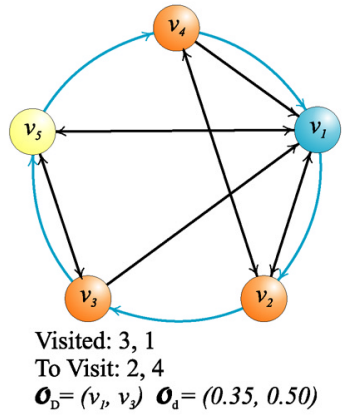

(d)

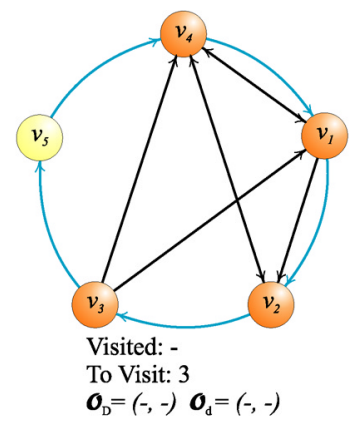

(b)

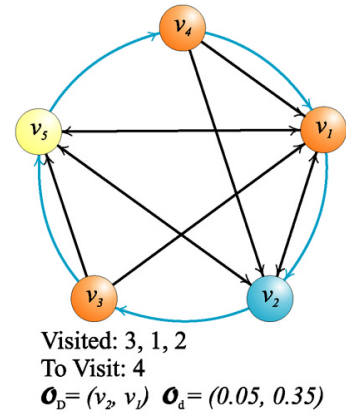

(e)

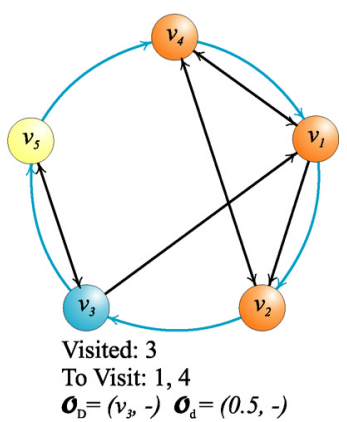

(c)

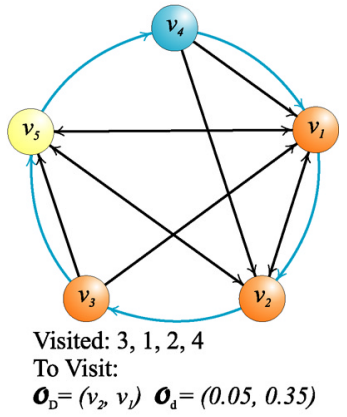

(f)

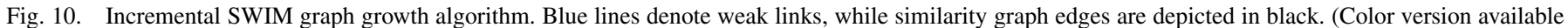
online at http://ieeexplore.ieee.org)

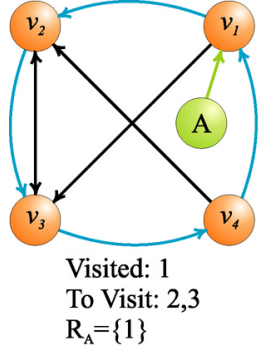

(a)

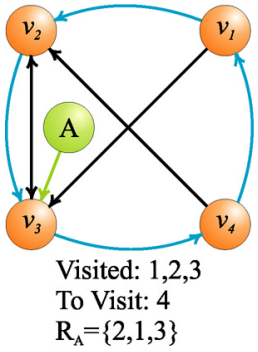

(c)



(b)

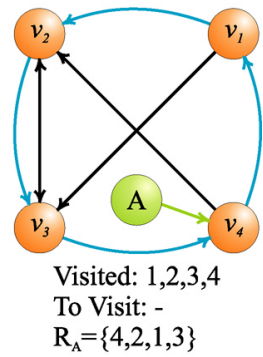

(d)
Fig. 11. SWIM agent search example where the query-by-example data is equal to the description of image $I_{4}$ at node four, or $\vec{d}\left(I_{4}\right)$. In (a), the agent A visits the seed node $v_{1}$ and obtains knowledge of nodes $v_{2}$ and $v_{3}$. The algorithm ends in (d) where all SWIM nodes have been traversed, and the ordered results set $R_{A}$ is complete. (Color version available online at http://ieeexplore.ieee.org)

can be very different, and thus, they do not necessarily have to exist on one computer system. Unlike conventional, centralized indexes which are simply collections of data describing a large storehouse of sedentary images, the SWIM paradigm empowers images by allowing them to become active entities in highly networked environments. Each SWIM node exists as its own entity, as its own agent, and as a member of a large network of
TABLE II

DisTANCE/INCIDENCE MATRIX FOR THE SWIM AGENT RETRIEVAL EXAMPLE OF FIG. 11 WHERE $F=1$, BOLDFACE ENTRIES REPRESENT INDICES FOR SOURCE(ROW)-TARGET(COLUMN) EDGES

\begin{tabular}{c||c|c|c|c}
\hline & $v_{1}$ & $v_{2}$ & $v_{3}$ & $v_{4}$ \\
\hline$v_{1}$ & 0 & $\mathbf{0 . 4}$ & 0.3 & 0.4 \\
\hline$v_{2}$ & 0.4 & 0 & $\mathbf{0 . 2}$ & 0.35 \\
\hline$v_{3}$ & 0.3 & $\mathbf{0 . 2}$ & 0 & 0.6 \\
\hline$v_{4}$ & 0.4 & $\mathbf{0 . 3 5}$ & 0.6 & 0 \\
\hline
\end{tabular}

other similar entities which through their interactions, form a self-organized small world.

A number of experiments can be performed into order to measure system capabilities but because of the fact that the Small World Indexing Model follows a distributed paradigm, it is very important that the benefits of distributed computing be demonstrated here. The key characteristic which we want to address is that of fault tolerance in terms of graceful system performance degradation in the presence of component failure. In the case of a centralized index, problems associated with any single component can result in a system-wide failure, rendering the entire index inaccessible. It is demonstrated that the failure of both individual SWIM nodes and links to SWIM nodes result in a graceful decay of overall system performance. This ability of the SWIM index to deteriorate slowly in light of component failure is beneficial in a modern ubiquitously networked environment where the extremely high number of potential global users accessing a database means that any system downtime can translate to a loss in revenue.

\section{A. Experimental Results}

In performing a resiliency analysis, an image database consisting of 4281 general images was employed. A SWIM network 


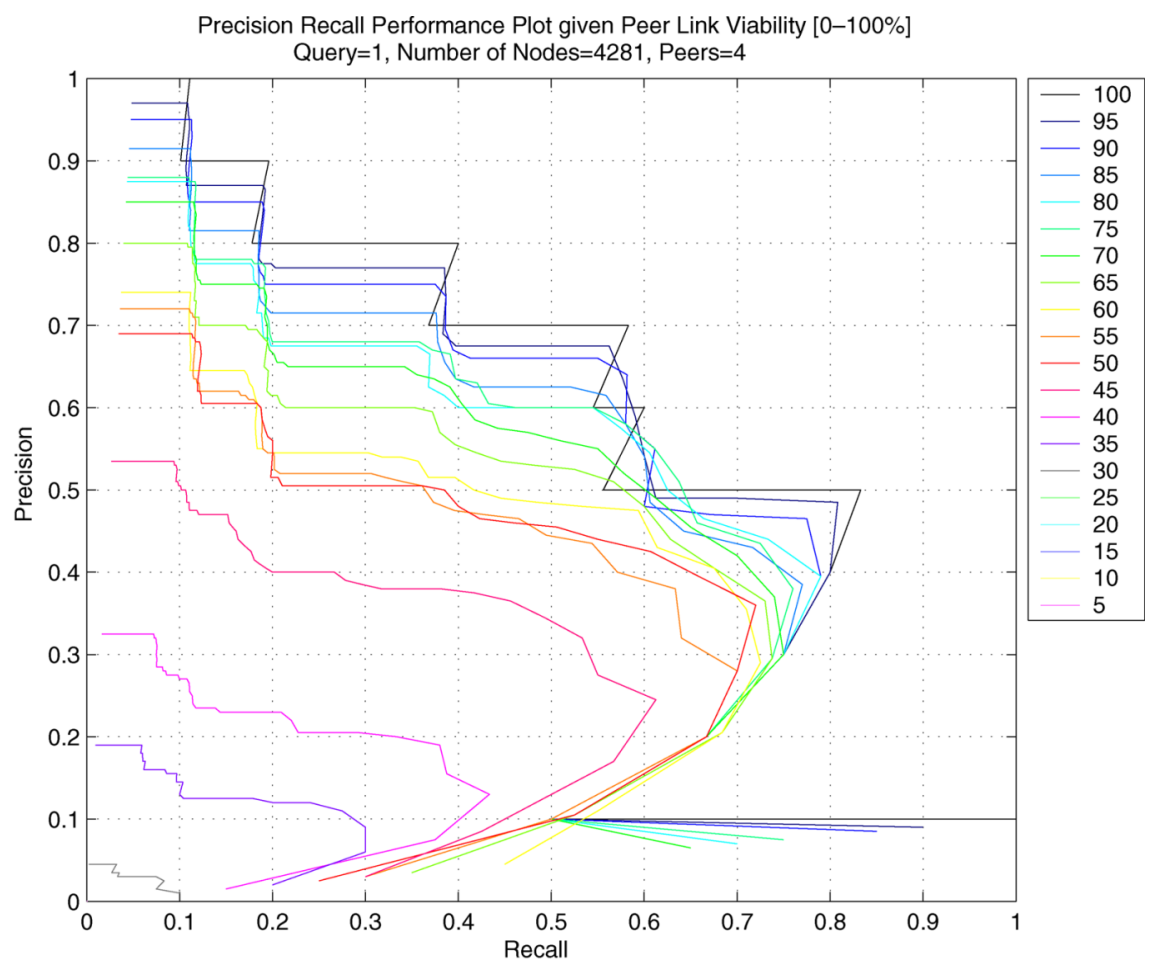

(a)

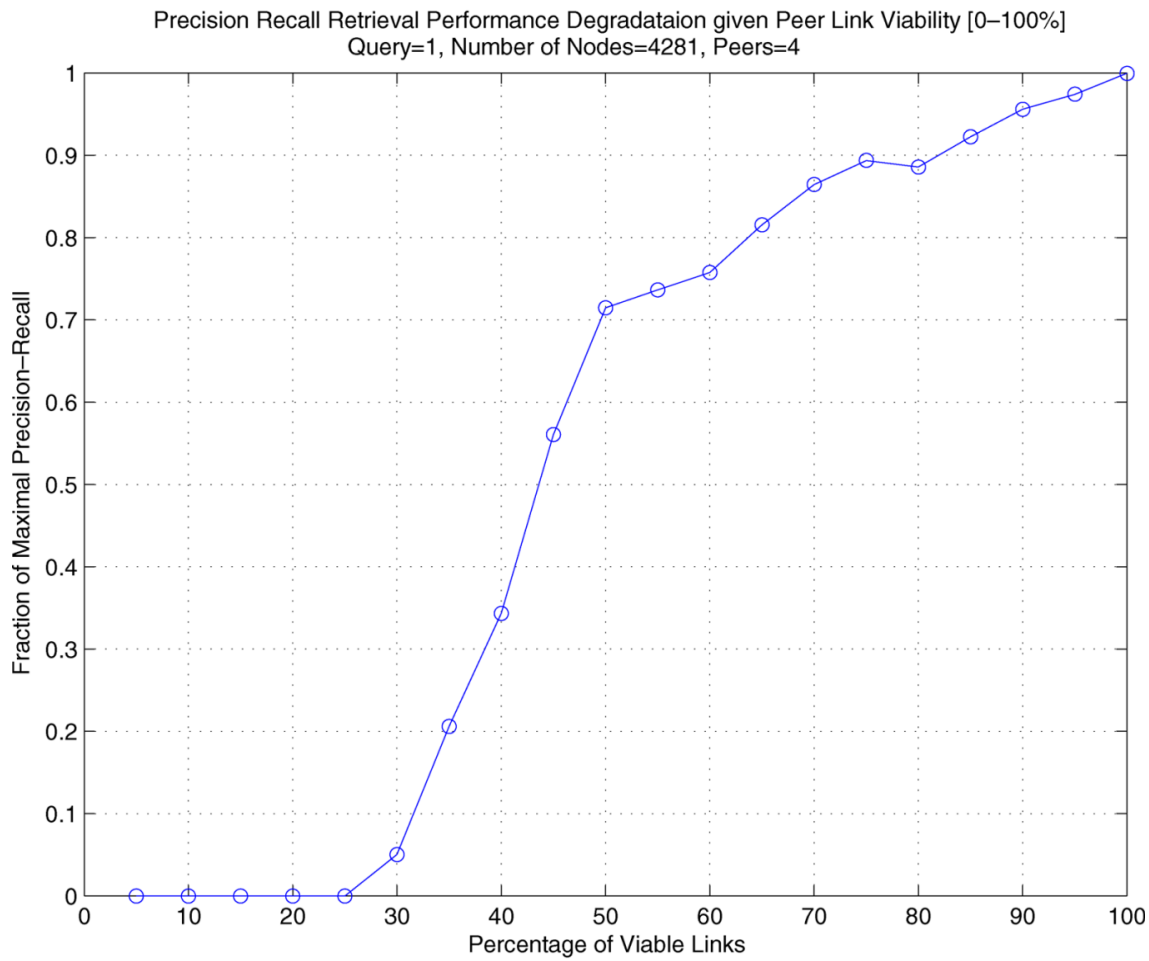

(b)

Fig. 12. Degradation of the precision-recall performance for query 1 is shown in (a) as the number of viable links in the SWIM network is decreased. The rate of decrease is illustrated in (b) as a percentage of maximum. (Color version available online at http://ieeexplore.ieee.org)

using the Color Structure descriptor with the number of peers equal to four was generated, and then both links and nodes were randomly removed from the network before proceeding with searches. In addition, a Euclidean distance was used for performing similarity calculations between image descriptions. For the removal of single links, a uniform random variable was em- ployed. A uniform random variable was also used in the removal of SWIM nodes from the overall topology. This was achieved through the nullification of all connections incident to and incident from a deleted node (thus making it unreachable from its peers). In both cases, no distinction was made between edges from the similarity graph and edges from the weak graph. Once 


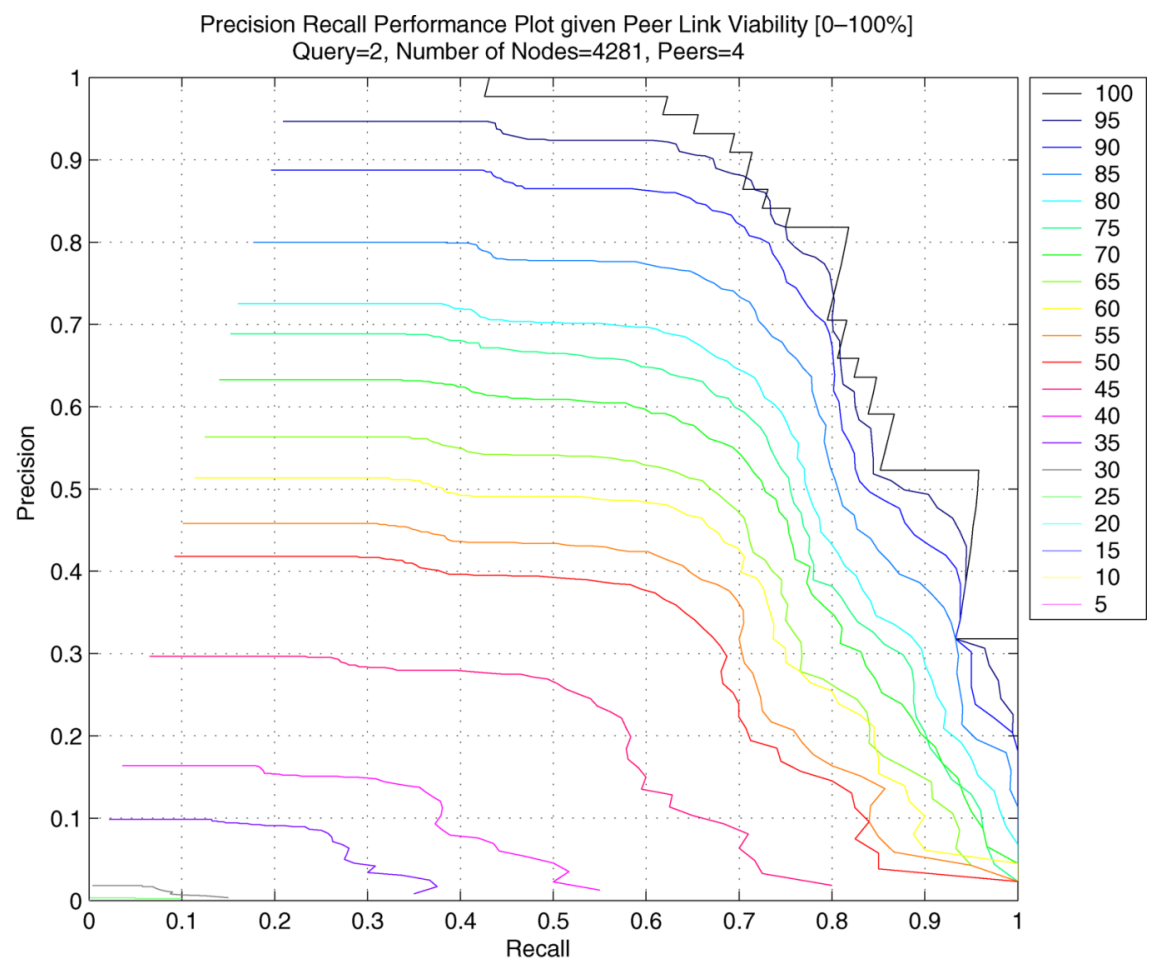

(a)

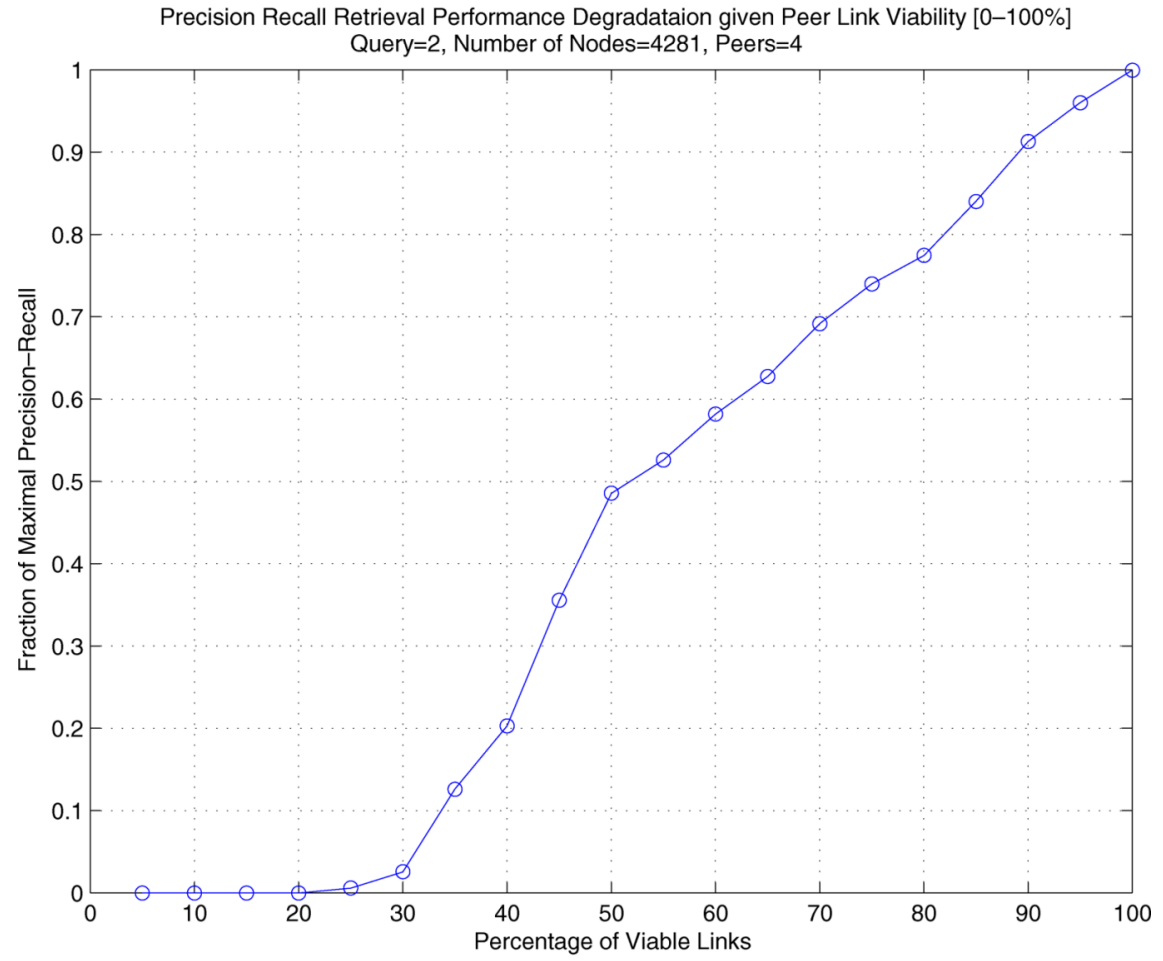

(b)

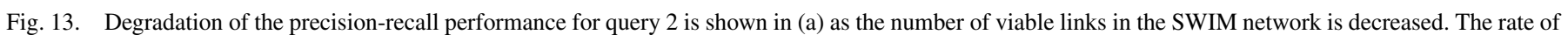
decrease is illustrated in (b) as a percentage of maximum. (Color version available online at http://ieeexplore.iee.org)

the required number of nodes or links had been removed from the system, a retrieval agent [5] was introduced into the system at a randomly assigned SWIM node and instructed to perform a complete search of the entire SWIM network. Upon completion of the search, a precision-recall analysis was done to observe the effect of network failures on the retrieval performance.
The random removal of links from the SWIM network was performed by first considering the total number of links in the overall topology and then removing a percentage in 5\% increments. In this case, the SWIM realization used four peers for a total of five outgoing links per node (4 peers +1 weak link). Since the total number of images in the SWIM network was 
equal to 4281, a total of 21405 links were available for removal. The precision-recall plots of Figs. 12(a) and 13(a) illustrate the degradation of retrieval performance for two specific queries. As can be seen in all the precision-recall plots, 20 different curves are shown for the 20 different cases of the total number of valid peer links. The best performance is achieved when $100 \%$ of the links are valid and the worst performance occurs when only $5 \%$ of the links are valid. To remove bias that may have been introduced from inserting the search agent into the SWIM network from a specific node, each curve was averaged over one-thousand iterations where the agent's insertion point was randomly chosen from the set of remaining viable nodes. Since the maximal performance of the retrieval agent is already known, the retrieval data from each successively degraded network was compared to the ideal case in order to create an overall performance degradation curve (see associated (b) plots for Figs. 12 and Fig. 13). Because precision-recall plots are parameterized according to the number of retrievals $k$, a Euclidean distance between curves was calculated in achieving these overall degradation curves.

\section{CONCLUSIONS}

This paper introduced and described an indexing and retrieval scheme based on small world theory. This methodology creates the foundation of a distributed image database system that uses agents to insert data and also to perform searching. The underlying mechanism is based on storing similarity information of a given image node and of other nodes which may exist anywhere on a network. This provides a distributed infrastructure that proves to be resilient and tolerant to faults. Since the Small World Indexing Mine treats each image as an independent host in a P2P network it is subject to link failures, experiments were performed to examine the resiliency of SWIM indexed images to problems related to the validity of its internal peer connections. This was performed by randomly invalidating peer links, and then performing agent-based retrievals to determine the system's overall capacity for serving results to a user. The continuous deletion of links resulted in the SWIM network exhibiting a correspondingly graceful degradation of performance. Thus, the SWIM network demonstrates an overall ability to still maintain partial functionality in the presence of component failures. In light of this, it is can be said that the use of the SWIM network for distributed image indexing and retrieval is superior to a centralized approach, which due to its nature, is unable to provide gracefully deteriorating and partial levels of service when one of its components fail.

\section{ACKNOWLEDGMENT}

The authors would like to thank the reviewers for their thorough comments and suggestions that helped to improve this paper.

\section{REFERENCES}

[1] W. Niblack, R. Barber, W. Equitz, M. Flickner, E. Glasman, D. Petkovic, P. Yanker, C. Faloutsos, and G. Taubin, "The QBIC project: Querying images by content using color, texture and shape," in Proc. SPIE, Storage and Retrieval for Image and Video Databases, vol. 1908, San Jose, CA, 1993, pp. 173-187.
[2] R. Brunelli and O. Mich, "COMPASS: An image retrieval system for distributed databases," in Proc. IEEE Int. Conf. Multimedia, vol. 1, Jul. 2000, pp. 145-148.

[3] C. H. Ng and K. C. Sia, "Peer clustering and firework query model," in Proc. 11th World Wide Web Conf., Honolulu, HI, 2002.

[4] I. Stoica, R. Morris, D. Liben-Nowell, D. Karger, M. F. Kaashoek, F. Dabek, and H. Balakrishnan, "CHORD: A scalable, peer-to-peer lookup service for internet applications," in Proc. ACM SIGCOMM, Aug. 2001.

[5] P. Androutsos, D. Androutsos, A. Kushki, K. N. Plataniotis, and A. N. venetsanopoulos, "Distributed MPEG-7 image indexing using small world user agents," in Proc. IEEE Int. Conf. Image Processing (ICIP), Singapore, Oct. 2004.

[6] I. S. Pool and M. Kochen, "Contacts and influence," Social Networks, vol. 1 , no. 1 , pp. $1-51,1978$.

[7] S. Milgram, "The small world problem," Psychol. Today, vol. 2, pp. 60-67, 1967.

[8] J. Guare, Six Degrees of Separation: A Play New York, 1990.

[9] D. O'Brien, "Trivia Game Finds Bacon at the Heart of the Acting Universe," The Chicago Tribune, p. 1, 3, Jul. 8, 1996.

[10] The Oracle of Bacon at Virginia [Online]. Available: http://www.cs.virginia.edu/oracle/

[11] J. Kleinberg and S. Lawrence, "The structure of the web," Science, vol. 30, no. 294, pp. 1849-1850, Nov. 2001.

[12] L. A. Adamic, "The small world web," in Proc. Eur. Conf. Digital Libraries (ECDL '99), vol. 1696, Lecture Notes in Computer Science, 1999.

[13] A.-L. Barabási, R. Albert, and H. Jeong, "Scale-free characteristics of random networks: The topology of the world-wide web," Physica A, vol. 272, pp. 173-187, 1999.

[14] R. Albert, H. Jeong, and A.-L. Barabási, "Diameter of the world-wide web," Nature, vol. 401, pp. 130-131, 1999.

[15] A. Brodeur, R. Kumar, F. Maghoul, P. Raghavan, S. Rajagopala, R. Strata, A. Tomkins, and A. Wiener, "Graph structure of the web," Comput. Networks, vol. 33, pp. 309-320, 2000.

[16] B. Bollobás, C. Borgs, J. Chayes, and O. Riordan, "Directed scale-free graphs," in Proc. 14th ACM-SIAM Symp. Discr. Alg., 2003, pp. 132-139.

[17] D. J. Watts and S. H. Strogatz, "Collective dynamics of 'small-world" networks," Nature, vol. 393, pp. 440-442, 1998.

[18] P. Erdós and A. Rényí, "On random graphs," Publ. Math., vol. 6, pp. 290-297, 1959.

[19] M. E. J. Newman and D. J. Watts, "Scaling and Percolation in the SmallWorld Network Model," Santa Fe Institute, Working Paper 99-05-034, 1999.

[20] A.-L. Barabási and R. Albert, "Emergence of scaling in random networks," Science, vol. 286, pp. 509-512, 1999.

[21] M. E. J. Newman, "Models of the Small World," Santa Fe Institute, Working Paper \#99-12-080, 1999.

[22] S. Chang, T. Sikora, and A. Puri, "Overview of the MPEG-7 standard," IEEE Trans. Circuits Syst. Video Technol., vol. 11, pp. 688-695, 2001.

[23] M. Granovetter, "The strength of weak ties," Amer. J. Sociology, vol. 78, no. 6, pp. 1360-1380, May 1973.

[24] M. S. Granovetter, "The strength of weak ties: A network theory revisited," Sociological Theory, vol. 1, pp. 201-233, 1983.

[25] S. Antani, R. Kasturi, and R. Jain, "A survey of the use of pattern recognition methods for abstraction, indexing and retrieval of images and video," Pattern Recognit., vol. 35, pp. 945-965, 2002.

[26] S. Santini and R. Jain, "Similarity measures," IEEE Trans. Pattern Anal. Mach. Intell., vol. 21, no. 9, pp. 871-883, 1999.
Panagiotis Androutsos (M'03) received the B.A.Sc., M.A.Sc., and Ph.D. degrees, all from the Department of Electrical and Computer Engineering, University of Toronto, Toronto, ON, Canada in 1997, 1999, and 2004, respectively.

$\mathrm{He}$ is currently an Imaging Researcher and Software Developer with the Dominion Voting Systems Corporation, Toronto. His interests lie in distributed image databases, multimedia databases, image analysis, and image processing. 
Dimitrios Androutsos (SM'05) received the B.A.Sc., M.A.Sc., and Ph.D. degrees, all from the Department of Electrical and Computer Engineering, University of Toronto, Toronto, ON, Canada in 1992, 1994, and 1999, respectively.

$\mathrm{He}$ is currently an Associate Professor, Department of Electrical \& Computer Engineering, Ryerson University, Toronto. His research interests lie in the areas of image and video processing, multimedia archiving and retrieval, distributed multimedia databases, image and video compression, object segmentation, object tracking, image enhancement, and image filtering.

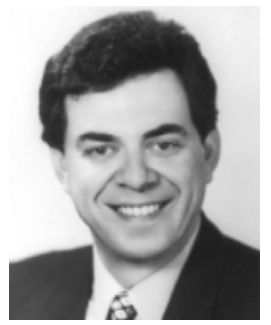

Anastasios N. Venetsanopoulos (SM'79-F'88) received the Diploma in engineering degree from the National Technical University of Athens (NTU), Athens, Greece, in 1965, and the M.S., M.Phil., and $\mathrm{Ph} . \mathrm{D}$. degrees in electrical engineering from Yale University, New Heaven, CT, in 1966, 1968, and 1969 , respectively.

He joined the Department of Electrical and Computer Engineering of the University of Toronto, ON, Canada, in September 1968, as a Lecturer and he was promoted to Assistant Professor in 1970, Associate Professor in 1973, and Professor in 1981. Since July 1997, he has been Associate Chair: Graduate Studies of the Edward S. Rogers Sr. Department of Electrical and Computer Engineering and was Acting Chair during the spring term of 1998-1999. Since July 2001, he has served as the 12th Dean of the Faculty of Applied Science and Engineering of the University of Toronto. He has served as Chair of the Communications Group and Associate Chair of the Department of Electrical Engineering and Associate Chair: Graduate Studies for the Department of Electrical and Computer Engineering. He was on research leave at Imperial College of Science and Technology, the National Technical University of Athens, the Swiss Federal Institute of Technology, the University of Florence and the Federal University of Rio de Janeiro, and has also served as Adjunct Professor at Concordia University. He has served as lecturer in 138 short courses to industry and continuing education programs and as Consultant to numerous organizations; he is a contributor to twenty eight (28) books, a coauthor of Color Image Processing and Applications (New York: Springer-Verlag, 2000), Nonlinear Filters in Image Processing: Principles Applications (Norwell, MA: Kluwer, 1990), Artificial Neural Networks: Learning Algorithms, Performance Evaluation and Applications (Norwell, MA: Kluwer, 1993), and Fuzzy Reasoning in Information Decision and Control systems (Norwell, MA: Kluwer 1994). He has served as Chair on numerous boards, councils and technical conference committees of the IEEE, such as the Toronto Section (1977-1979) and the IEEE Central Canada Council (1980-1982); he was President of the Canadian Society for Electrical Engineering and Vice President of the Engineering Institute of Canada (EIC) (1983-1986). He was a Guest Editor or Associate Editor for several IEEE Journals and the Editor of the Canadian Electrical Engineering Journal (1981-1983). He was the Technical Program Co-Chair of the IEEE International Conference on Image Processing (ICIP'01). He has published 750 papers in refereed journals and conference Proceedings on digital signal and image processing, and digital communications.

Prof. Venetsanopoulos is a member of the IEEE Communications, Circuits and Systems, Computer, and Signal Processing Societies, as well as a member of Sigma Xi, the Technical Chamber of Greece, the European Association of Signal Processing, the Association of Professional Engineers of Ontario (APEO) and Greece. He was elected as a Fellow of the IEEE "for contributions to digital signal and image processing." He is also a Fellow of the EIC, "for contributions to electrical engineering," and was awarded an Honorary Doctorate from the National Technical University of Athens, in October 1994. In October 1996, he was awarded the "Excellence in Innovation Award" of the Information Technology Research Center of Ontario and Royal Bank of Canada, "for innovative work in color image processing." 